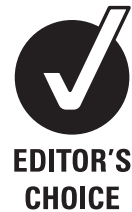

${ }^{1}$ Centre for Research into Disability and Society within the Curtin Health Innovation Research Institute, Curtin University of Technology, Perth, Australia ${ }^{2}$ Centre for Research on Ageing within the Curtin Health Innovation Research Institute, Curtin University of Technology, Perth, Australia ${ }^{3}$ Association for the Blind of Western Australia, Perth, Australia ${ }^{4}$ School of Exercise, Biomedical and Health Sciences, Edith Cowan University, Perth, Australia ${ }^{5}$ School of Public Health, Curtin Health Innovation Research Institute, Curtin University of Technology, Perth, Australia ${ }^{6}$ School of Occupational Therapy, Dalhousie University, Halifax, Canada

\section{Correspondence to}

Professor Tanya L Packer, Curtin Health Innovation Research Institute, Faculty of Health Sciences, GPO Box U1987 Perth, Australia 6845;

t.packer@curtin.edu.au

Accepted 26 June 2009

\title{
Vision self-management for older adults: a randomised controlled trial
}

\author{
Sonya J Girdler, $1,2,3,4$ Duncan P Boldy, ${ }^{2,5}$ Satvinder S Dhaliwal, ${ }^{1,2,5}$ \\ Margaret Crowley, ${ }^{1,3}$ Tanya L Packer ${ }^{1,3,6}$
}

\section{ABSTRACT}

Background/aims Ageing of the population will result in unprecedented numbers of older adults living with agerelated vision loss (ARVL). Self-management models improve health outcomes and reduce healthcare costs; however, the principles have rarely been applied in low vision services.

Methods A two-armed randomised controlled trial of older adults $(n=77)$ with ARVL compared 'usual care' provided by a not-for-profit community agency with an extended model of care (usual care + self-management group intervention). The primary outcome variable (participation in life situations) was measured using the Activity Card Sort. Secondary outcome measures examined general health and vision-specific domains.

Results The intention-to-treat analysis demonstrated that the extended model produced significantly better participation in life situations at post-test when compared with the usual care only group. Gains were made regardless of whether participants were, or were not, depressed at baseline. The addition of the selfmanagement group was also successful in significantly reducing depression, increasing physical and mental health, generalised and domain-specific self-efficacy, and adjustment to ARVL. With the exception of adjustment and mental health, differences were still apparent at 12 weeks' follow-up.

Conclusion Addition of self-management significantly improved general health and vision-specific rehabilitation outcomes for older adults with ARVL.

\section{INTRODUCTION}

Nearly half a million Australians have impaired vision. With prevalence trebling with each decade over age 60, this figure is projected to rise to 800000 by $2024 .{ }^{1}$ In 2004 , the direct costs were estimated at \$A1.8 billion, indirect costs at \$A3.2 billion and the cost of suffering and premature death a further $\$ A 4.8$ billion. In spite of critical advances in the medical management of ocular pathology, many people experience age-related vision loss (ARVL) that is non-correctable. With a growing body of research documenting negative impacts of ARVL on the psychological, social and daily functioning of older adults, ${ }^{23}$ effective interventions are needed.

Based on landmark qualitative research, ${ }^{4}$ selfmanagement programmes assist people in managing their disease/symptoms, as well as the emotional and daily consequences of living with a chronic condition. Use of self-management in low vision services is limited, but a recent systematic review of education programmes for macular degeneration found three protocols reported in four studies ( $\mathrm{n}=532$ ) with three follow-up studies. Although all contained elements of self-management, only one was explicitly described as such. Effect sizes for the outcomes ranged from small to very large (0.14 to 1.21). The specific self-management programme was reported in two controlled trials and a follow-up study. ${ }^{5-7}$ In the first trial, participation was effective in decreasing depression and anxiety, increasing domain-specific self-efficacy and use of low vision aides in comparison with a wait-list control group $(n=92) .{ }^{5}$ In the second trial $(n=232)$, participation significantly reduced emotional distress and improved everyday functioning as compared with two control groups $(12 \mathrm{~h}$ of tape-recorded health information and a wait-list control). ${ }^{6}$ Six-month follow-up revealed the programme to be protective against development of clinical depression. ${ }^{7}$ The systematic review concluded, however, that there was a need for studies with more robust methodology including an intent-to-treat analysis. ${ }^{8}$ The primary objective of this RCT was to evaluate the differential effects of usual care (UC) and usual care plus vision selfmanagement (UC+VSM) on health and participation outcomes of older adults with ARVL. It was judged unethical to withhold UC; hence a notreatment control group was not included. The secondary objective was to examine the impact of depression at pretest on the outcome variables.

\section{MATERIALS AND METHODS}

A two-armed RCTevaluated UC in comparison with $\mathrm{UC}+\mathrm{VSM}$, both provided by the Association for the Blind of Western Australia (ABWA), a not-for-profit community low vision agency. ABWA provides vision rehabilitation services to over 2000 Western Australians annually who are blind or vision impaired. Data collection occurred at baseline, immediately after the completion of rehabilitation and 12 weeks later (follow-up) (figure 1). Face-toface structured interviews (including oral administration of questionnaires) were administered in participants' homes. Ethical approval was obtained from the Curtin University of Technology Human Research Ethics Committee and the Chief Executive Officer of ABWA. All participants provided informed consent and (1) were aged 65 or over, (2) were diagnosed as having ARVL by an ophthalmologist, (3) had best corrected vision at the Snellen equivalent of $6 / 12$ or less in both eyes, (4) were living in independent accommodation in the community, (5) had sufficient physical stamina, mental functioning, hearing and communication ability to attend the group intervention, and 6) were newly referred to the agency or had recontacted the 
Figure 1 Flow of participants through the trial.

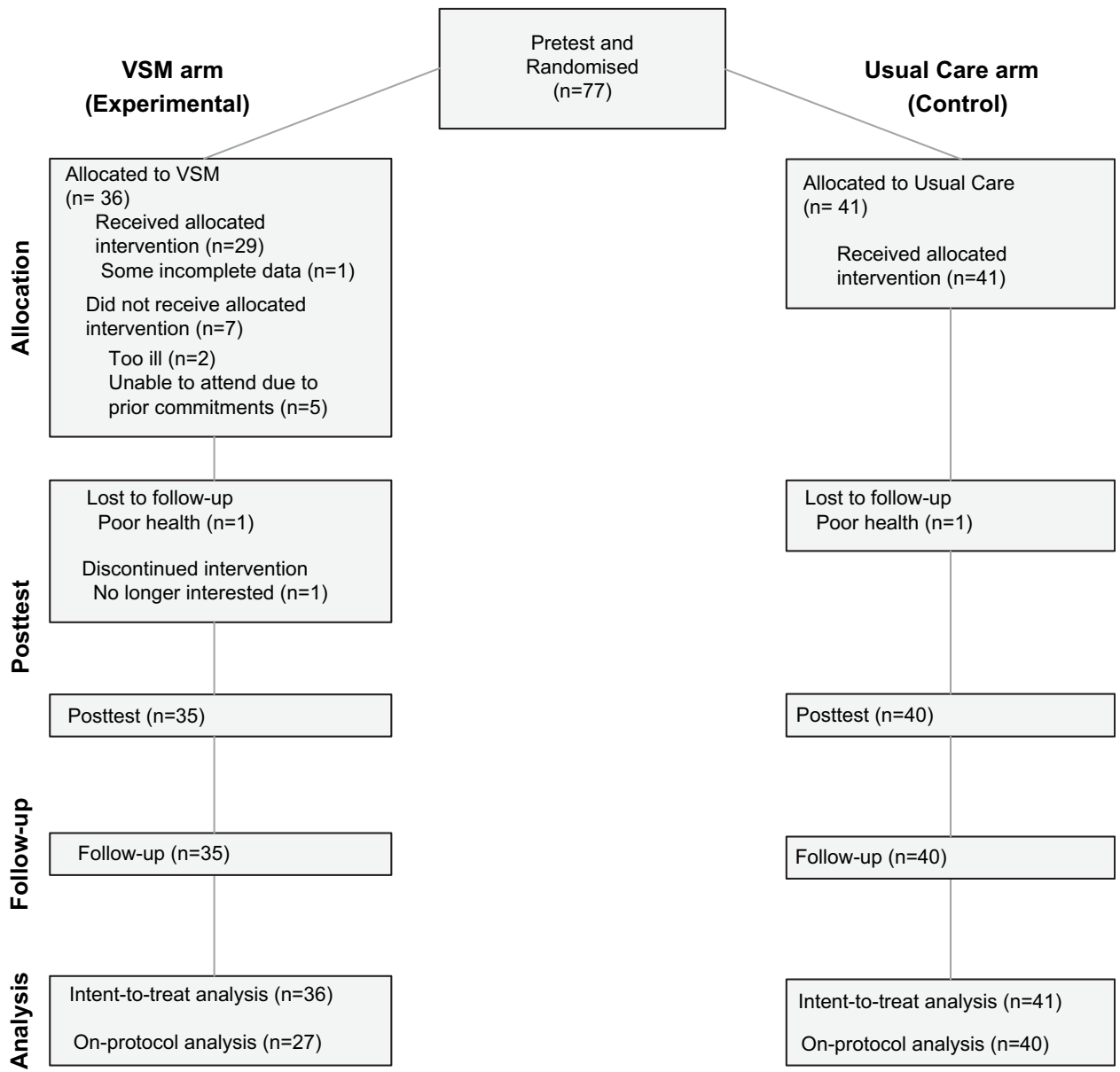

agency after a significant deterioration in their vision (defined as 'need for new low vision aids'). Allocation was by random assignment via computer-generated random numbers table. Socio-demographic data and participant clinical characteristics were collected at baseline. The independent variable was intervention type (UC or UC+VSM) (table 1). For details of the needs assessment, see Girdler et $a l^{9}$ and details of the intervention see Packer et al. ${ }^{10}$

\section{Participation}

The original Activity Card Sort (ACS) ${ }^{11}$ was used to measure the primary outcome variable-participation levels. Images and descriptions of everyday activities were digitally magnified on a laptop computer. A standardised description of each image was read to participants unable to see the screen. Test-retest reliability $(r=0.90)$ has been reported in a sample of 20 community dwelling older adults, ${ }^{11}$ and construct validity has been established in various samples of older adults. ${ }^{12}$ Current activity level as outlined in the Test Manual was calculated.

\section{Depression}

Developed for use with people over the age of 60, the widely used 30-item Geriatric Depression Scale (GDS) includes fewer somatic items than other scales. ${ }^{13}$ It has excellent internal consistency ( $\boldsymbol{\alpha}=0.94)$, 1-week test-retest reliability $(r=0.85)$ and concurrent validity with the Zung Self-Rating Depression Scale $(\mathrm{r}=0.83)$ and the Hamilton Rating Scale for Depression $(\mathrm{r}=0.84) .{ }^{14}$

\section{Quality of life}

Quality of life was measured with the Australia/New Zealand version of the SF-36 Health Survey (Version 1.0). ${ }^{15}$ Physical Component Summary (PCS) and Mental Component Summary (MCS) scores were calculated $^{16}$ using a customised SAS/STAT programme based on Australian normative data (mean $=50, \mathrm{SD}=10) .{ }^{17}$

\section{Generalised self-efficacy}

Participants' strength of belief in ability to manage a wide range of everyday problems and difficulties was measured with the Generalised Self-Efficacy Scale (GSES). ${ }^{18}$ Schwarzer $^{18}$ demonstrated the unidimensionality of the scale, a high level of internal

Table 1 Key features of the interventions

\begin{tabular}{ll}
\hline Usual care & Usual care + vision self-management programme \\
\hline One-to-one case management model & 'Usual care,' plus self-management: \\
Initial interview in client's home by service coordinator to discuss service needs & Based on local qualitative study, included self-management, self-efficacy and \\
and to jointly develop service plan & group model of service delivery theories and principles \\
Visual assessment at low-vision clinic by optometrists and orthoptists; & revision of homework, learning sessions (including learning and practice activities) \\
prescription of low-vision aids; aids provided on a trial basis. & and homework assignments \\
Referral to internal (occupational therapy, orthopty, social work, orientation and & Delivered in a group environment with six to 10 participants, and led by an \\
mobility training, low-vision technology) and external service providers & occupational therapist and a social worker
\end{tabular}


consistency $(\alpha=0.93)$, and the absence of gender bias in mean scale scores in a sample $(n=249)$ of older adults. The English version has been supported by Barlow et al, ${ }^{19}$ who reported a high internal consistency $(\boldsymbol{\alpha}=0.88)$ and test-retest reliability of 0.63 .

\section{Adaptation}

The Adaptation to Vision Loss Scale (AVLS) ${ }^{20}$ is a measure of adaptation to vision loss, low visual rehabilitation (LVR) and relationships with family and friends. It has a good internal consistency $(\boldsymbol{\alpha}=0.84)$ and reliability $(\boldsymbol{\alpha}=0.83) .{ }^{21}$ It is sufficiently sensitive to record positive changes following provision of vision rehabilitation services. ${ }^{21}$

\section{Domain-specific self-efficacy}

Vision-specific self-efficacy was measured using the Macular Degeneration Self-Efficacy Questionnaire (AMD-SEQ) ${ }^{5}$ adapted (with permission) to reflect generic ARVL (ARVL-SEQ). The AMD-SEQ is internally consistent ( $\alpha$ ranging from 0.60 to 0.74 ) and reliable over 2 days $(r=0.70$ to 0.88$)$ and 6 weeks $(r=0.59$ to $0.89) .{ }^{5}$ It is sufficiently sensitive to record positive change at posttest and 6 months following participation in a self-management intervention. ${ }^{67}$

Using pilot data, ${ }^{10}$ a power calculation for analysis of variance (80\% power and $5 \%$ significance) determined the need for 38 participants per group. Statistical analyses were performed using SPSS version 12.0.1 (SPSS, Chicago). All analyses, unless otherwise stated, were conducted on an intention-to-treat basis ${ }^{22}$ (see figure 1). Missing data were imputed using either carry forward or mean substitution.

Baseline socio-demographic and clinical characteristics of the two groups were compared using independent sample $t$ tests for continuous variables, $\chi^{2}$ tests for independence and Fisher exact tests for categorical variables. Analysis of covariance (ANCOVA) with pretest scores as covariate examined effectiveness (primary and secondary outcomes). Normality and homogeneity of variance were tested and, where necessary, data transformations performed. Effect size was measured using partial eta squared and was interpreted based on Cohen's proposed conventional values for analysis of covariance: small $=0.10$, medium $=0.3$ and large $=0.50 .^{23}$ The number of participants depressed (GDS $\geq 11$ ) versus not depressed in each group was compared at each data collection point using the $\chi^{2}$ test for independence. Statistical significance for all tests was set a priori at 0.05 .

To examine the interaction between the model of service delivery and clinically significant symptoms of depression at pretest on the primary outcome measure, a two-way ANCOVA was performed. The type of intervention and depression (yes/no based on a GDS $\geq 11$ ) were entered as fixed factors, pretest ACS scores as the covariate, and post-test and follow-up ACS scores as dependent variables.

\section{RESULTS}

Thirty-six participants were allocated to UC+VSM and 41 to UC (figure 1). Seven participants did not attend the self-management programme as allocated due to illness $(n=2)$ or prior commitments $(n=5)$. They were, however, included in the intent-totreat analysis. Of those who began the VSM programme as allocated $(n=29)$ attendance was high; five participants missed one session each, and one participant missed two. One participant was lost to follow-up in each group.

The random number generation model resulted in slightly different participant numbers in the two groups. There were no significant differences between groups on any measured characteristic. The mean age was 79.1 years $(S D=6.7)$, which reflects the age-related nature of visual impairment. Also reflective of ageing, only $18 \%$ of participants reported vision impairment as their sole medical condition. The primary cause of vision impairment, age-related macular degeneration, reflects that in an Australian population. ${ }^{24}$ Sixty-six per cent of participants rated their health as good, very good or excellent (table 2).

Participants in the experimental group had statistically better participation levels than the control group (table 3). Over the course of the study UC+VSM participants demonstrated an increase, then maintenance of participation in life situations, while UC participants experienced gradual decline. At post-test, those in the UC + VSM demonstrated a $5 \%$ increase in participation, while those receiving UC alone experienced a $5 \%$ decline (effect size $=0.20$ ); differences were sustained at 12 weeks follow-up $(p<0.001)$.

Secondary outcomes included general health measures (GDS, QOL and GSES) and vision-specific measures (AVLS and ARVL$\mathrm{SEQ}$ ). On all general health measures, the UC+VSM group demonstrated significantly better outcomes than the UC group at post-test, with differences retained at follow-up. On the GDS the adjusted pretest score (mean=10.58) was close to the 11 cutoff for clinically significant symptoms. In the experimental group, this dropped to 8.05 at post-test, with the $95 \%$ CI falling completely below the 11-point cut-off, both a clinically and a statistically significant improvement. In contrast, the mean score for the control group rose above the clinical cut-off (adjusted GDS mean=11.28). Analysis of depression as a dichotomous variable (depressed vs not depressed) revealed that at posttest, $51 \%$ of participants in the control group were depressed compared with only $36 \%$ of the UC+VSM participants. By 12 weeks' follow-up, only $17 \%$ of the experimental group (compared with $51 \%$ of controls) were experiencing symptoms of depression $\left(\chi^{2}=0.002, p=0.000\right)$.

At post-test, the UC+VSM participants also demonstrated significantly better physical and mental health (measured by the SF-36 PCS and MCS scores) and generalised self-efficacy (GSES) when compared with the control group ( $p \leq 0.019$ to 0.001 ). Scores remained largely unchanged from post-test to follow-up with UC+VSM participants continuing to demonstrate better outcomes. The one exception was mental health, where a slight improvement in scores of the UC group (SF-36 MCS) resulted in the loss of significance $(p=0.102)$ between the two groups. The extremely small effect size of 0.03 and a power of $36 \%$, suggests that this was related to statistical power.

On the two vision-specific measures (AVLS and ARVL-SEQ) post-test results again demonstrated better outcomes for the UC +VSM participants $(\mathrm{p} \leq 0.001)$ with medium effect sizes. An increase in scores of the UC group resulted in loss of significance $(\mathrm{p}=0.058)$ at follow-up.

To examine the interaction between type of service and presence of baseline depression scores, a two-way ANCOVA was performed: assumptions of normality, homogeneity of variance and reliability of measurement of the covariate were met. The independent variables were allocation (UC+VSM or usual care) and depression at pretest (depressed or non-depressed), and the dependent variable was participation in life situations (ACS) at post-test and follow-up. Baseline ACS scores were entered as a covariate. Results indicated a significant main effect for allocation at post-test $(F(1,73)=19.02, \mathrm{p}<0.001$, partial eta squared $=0.21)$ and at follow-up $(F(1,73)=32.79, \mathrm{p}<0.001$, partial eta squared=0.31). Participants in the UC+VSM had significantly higher levels of participation in life situations than those who received UC only at post-test and follow-up, irrespective of 
Table 2 Socio-demographic and clinical characteristics of all randomised participants at pretest

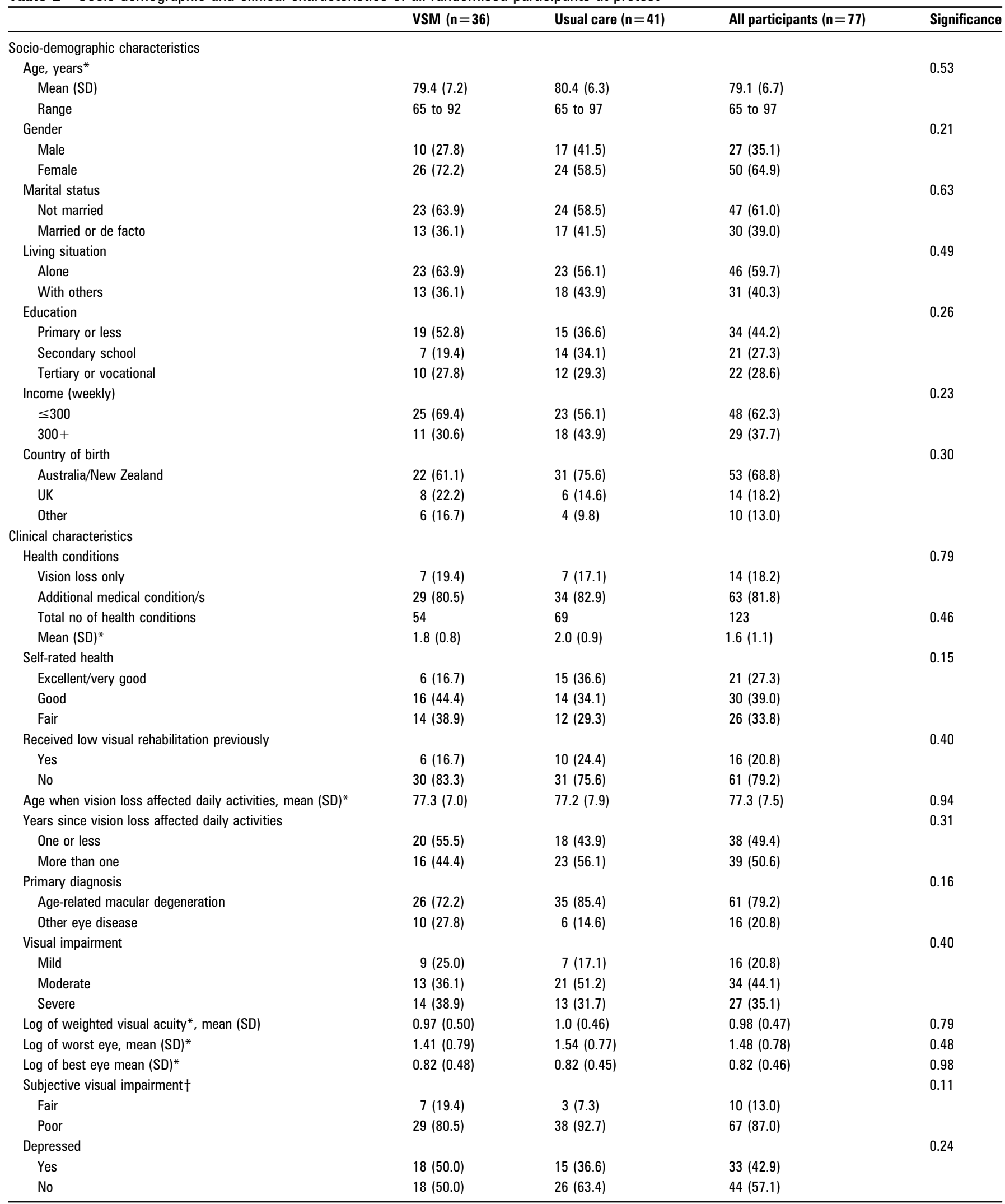

*Independent-samples $\mathrm{t}$ test.

†Fischer exact test.

whether or not they had clinically significant symptoms of depression at pretest. No statistically significant main effect for depression was found at either time (post-test $F(1,73)=0.07$, $\mathrm{p}=0.78$; follow-up $F(1,73)=0.05, \mathrm{p}=0.83$ ).

\section{DISCUSSION}

The addition of the self-management programme to UC resulted in better general and vision-specific outcomes. With the exception of adjustment to vision loss and mental health, these 
Table 3 Results of ANCOVA comparing usual care (UC) and usual care plus vision self-management (experimental group) (UC+VSM) and UC on primary and secondary outcomes at post-test and follow-up (pretest scores used as a covariate)

\begin{tabular}{|c|c|c|c|c|c|c|c|c|}
\hline \multirow[b]{2}{*}{ Measure } & \multirow[b]{2}{*}{ Group } & \multirow{2}{*}{$\begin{array}{l}\text { Pretest } \\
\text { Adjusted Mean }\end{array}$} & \multicolumn{3}{|c|}{ Post-test } & \multicolumn{3}{|c|}{ Follow-up } \\
\hline & & & Mean & Effect size $\neq$ & p Value & Mean & Effect size $\ddagger$ & p Value \\
\hline \multicolumn{9}{|l|}{ Primary outcome measure } \\
\hline \multirow[t]{2}{*}{$\mathrm{ACS}^{*}$} & $U C+V S M$ & 0.67 & 0.72 & 0.20 & 0.001 & 0.72 & 0.31 & 0.001 \\
\hline & UC & 0.67 & 0.62 & & & 0.56 & & \\
\hline \multicolumn{9}{|l|}{ Secondary outcome measures } \\
\hline \multicolumn{9}{|l|}{ General health domain } \\
\hline \multirow[t]{2}{*}{ Geriatric Depression Scale $†$} & $U C+V S M$ & 10.58 & 8.05 & 0.17 & 0.001 & 7.52 & 0.18 & 0.001 \\
\hline & UC & 10.58 & 11.28 & & & 10.83 & & \\
\hline \multicolumn{9}{|l|}{ SF-36 } \\
\hline \multirow[t]{2}{*}{ Physical Component Summary* } & UC+VSM & 36.07 & 38.16 & 0.10 & 0.005 & 38.86 & 0.23 & 0.001 \\
\hline & UC & 36.07 & 33.29 & & & 31.43 & & \\
\hline \multirow[t]{2}{*}{ Mental Component Summary* } & UC+VSM & 51.94 & 56.32 & 0.07 & 0.019 & 56.13 & 0.03 & 0.102 \\
\hline & UC & 51.94 & 51.71 & & & 53.49 & & \\
\hline \multirow[t]{2}{*}{ Generalised Self-Efficacy Scale* } & $\mathrm{UC}+\mathrm{VSM}$ & 29.56 & 31.55 & 0.20 & 0.001 & 31.08 & 0.14 & 0.001 \\
\hline & UC & 29.56 & 27.75 & & & 27.59 & & \\
\hline \multicolumn{9}{|l|}{ Vision-specific domain } \\
\hline \multirow[t]{2}{*}{ Adaptation to Vision Loss Scale* } & $\mathrm{UC}+\mathrm{VSM}$ & 52.67 & 61.16 & 0.27 & 0.001 & 62.10 & 0.05 & 0.058 \\
\hline & UC & 52.67 & 49.68 & & & 55.71 & & \\
\hline Age-related vision loss-Self-Efficacy & $\mathrm{UC}+\mathrm{VSM}$ & 54.10 & 78.81 & 0.30 & 0.001 & 80.14 & 0.30 & 0.001 \\
\hline Questionnaire & UC & 54.10 & 59.35 & & & 58.58 & & \\
\hline
\end{tabular}

*Increasing scores=Improvement.

†Decreasing scores $=$ Improvement.

$\neq$ Small $=0.10$, medium $=0.3$, large $=0.50 .^{23}$

differences were still apparent at 12 weeks' follow-up. Participation in life situations, as measured with the ACS, is of particular importance. The observed decline experienced by participants who received UC over the short period of the study (20-24 weeks) is alarming. The addition of self-management appears to not only arrest decline but also increase participation in life situations. This finding supports the assertion that participation in life situations is modifiable.

In the field of LVR, considerable debate surrounds the influence of depression on readiness for rehabilitation. ${ }^{3}$ While some have argued that rehabilitation should be delayed until depression has been treated, ${ }^{24}$ others have asserted that rehabilitation has a key role in reducing depression. ${ }^{7}$ Participants in the UC +VSM group increased their participation in life situations, irrespective of whether they reported clinically significant pretest symptoms of depression. UC+VSM was effective in reducing both the symptoms of depression and the number of participants with clinically significant symptoms, whereas UC alone was not, suggesting that the model of service delivery influences outcomes more than prerehabilitation depression.

The QOL of the two groups of participants demonstrated divergent trends. At both post-test and follow-up, the UC+VSM had significantly better mood, less psychological distress, and fewer social or role limitations due to emotional problems (SF 36 MCS). At follow-up, the UC+VSM participants, but not the UC group, reported PCS scores similar to community dwelling peers without vision impairment. ${ }^{17}$

At follow-up, both groups showed improved adaptation to vision loss, results similar to those from other research. ${ }^{20} 21$ However, the UC+VSM group appeared to adapt more quickly, with the two groups being significantly different at post-test. Others have also reported immediate benefits following participation in a group-based LVR. ${ }^{21}$

In the present study, the greatest magnitude of change reported by the UC+VSM group was domain-specific self-efficacy (ARVL$\mathrm{SEQ}$ ). In comparison, those who received UC demonstrated only small improvements. It is acknowledged that self-management programmes aim to increase self-efficacy; however, this dramatic increase accompanied by improvements in activity participation and depression levels further supports the assertion that selfefficacy is critical in mediating outcomes. ${ }^{7}$

Caution must be exercised when generalising findings. Participation was limited to community-dwelling older adults with ARVL within one geographical location, and all had chosen to seek LVR services. However, the sample appears to match the age, gender and vision diagnosis of older Australians. ${ }^{24}$ Selfselection bias is a common concern in behavioural interventions, but this reflects the reality of behavioural interventions in clinical practice. ${ }^{25}$ Although the assessor was masked to participants' group allocation, participants often inadvertently revealed their allocation during post-test and follow-up interviews. The quantitative self-report nature of outcomes reduced the potential bias to some extent. Finally, it is important to highlight that selfmanagement was provided in addition to usual care. It is possible that the effectiveness of the programme lies in the interaction between these two models of service delivery.

\section{CONCLUSION}

The present study has important strengths. Use of UC+VSM demonstrated clinically and statistically better health outcomes than UC alone. Importantly, the demonstrated declines experienced by the UC group were not seen in the UC+VSM group who made positive improvements. The conservative intentionto-treat approach adds confidence in the evidence of effectiveness demonstrated.

The present study demonstrated not only the effectiveness, but also the feasibility of delivering self-management programmes within a real service environment. Although conducting research in an 'in vivo' situation posed many challenges, it demonstrated that research in this field can be relevant and rigorous.

Acknowledgements The research was conducted in partnership with the Association for the Blind of Western Australia (Inc) and supported by 'in kind' contributions. The authors would like to acknowledge and sincerely thank the many people at the Association for the Blind (facilitators, health professionals and 
managers) whose commitment to clients and provision of quality care made this project possible. We would also like to thank the many people who volunteered to take part in this study: your efforts have improved the services to many others. We also wish to thank the staff at the ABWA who assisted with recruitment, facilitated the VSM programme and more generally embraced the opportunity to strive for excellence through rigorous research.

Funding This research was supported by an Australian Postgraduate Award, and a Freemasons Postgraduate Scholarship. This research was conducted in partnership with the Association for the Blind of Western Australia (Inc) and supported by 'in kind' contributions.

Competing interests ABWA purchases the services of the last author from Curtin University of Technology who holds the position of Director of Research, ABWA. The last author receives no personal gain from this arrangement.

Ethics approval Ethics approval was provided by the Curtin University of Technology Human Research Ethics Committee.

Provenance and peer review Not commissioned; externally peer reviewed.

\section{REFERENCES}

1. Acess Economics. Clear insight: the economic impact and cost of vision loss in Australia: an overview of the report prepared by Access Economics Pty Ltd. Melbourne: Centre for Eye Research Australia, University of Melbourne, 2004.

2. Brody B, Gamst A, Williams R, et al. Depression, visual acuity, comorbidity, and disability associate with age-related macular degeneration. Ophthalmology 2001:108:1893-900.

3. Horowitz A, Reinhardt JP, Boerner K. The effect of rehabilitation on depression among visually disabled older adults. Aging Ment Health 2005;9:563-70.

4. Corbin J, Strauss A. Unending work and care: managing chronic illness at home. San Francisco: Jossey-Bass Publishers, 1988.

5. Brody B, Williams R, Thomas R, et al. Age-related macular degeneration: a randomized clinical trial of a self-management intervention. Ann Behav Med 1999:21:322-9.

6. Brody B, Roch-Levecq A, Ganst A, et al. Self-management of age-related macular degeneration and quality of life. Arch Ophthalmol 2002;120:1477-83.

7. Brody B, Roch-Levecq A, Thomas R, et al. Self-management of age-related macular degeneration at the 6-month follow-up: a randomized controlled trial. Arch Ophthalmol 2005;123:46-53.
8. Lee L, Packer TL, Tang SH, et al. Self-management education programs for age-related macular degeneration: a systematic review. Australas J Ageing 2008;27:170-6.

9. Girdler S, Packer TL, Boldy D. The impact of age-related vision loss: a focus group study. OTJR: Occupation, Participation and Health 2008;28:110-20.

10. Packer TL, Girdler S, Boldy DP, et al. Vision self-management for older adults: a pilot study. Disabil Rehabil 2009;31:1353-61.

11. Baum C, Edwards D. Activity card sort: test manual. St Louis: Washington University School of Medicine, 2001.

12. Baum CM. The contribution of occupation to function in persons with Alzheimer's disease. J Occup Sci 1995;2:55-67.

13. Yesavage JA, Brink TL, Rose TL, et al. Development and validation of a geriatric depression screening scale: a preliminary report. J Psychiatr Res 1983;17:37-49.

14. Corcoran K, Fischer J. Measures for clinical practice: a sourcebook. 2nd rev edn. New York: The Free Press, 2000.

15. Health Assessment Lab. International Quality of Life Assessment Project Australia/New Zealand translation of the SF-36 Health Survery (Version 1.0). Boston: QualityMetric Inc, 1994.

16. Ware J, Kosinski M. SF-36 Health Survey: physical \& mental health summary scales: a manual for users of Version 1. Lincoln (RI): QualityMetric Inc, 2001.

17. Australian Bureau of Statistics. National Health Survey: SF-36 population norms Australia. Sydney: Australian Bureau of Statistics, 1995.

18. Schwarzer R. Measurement of perceived self-efficacy: psychometric scales for crosscultural research. Berlin: Free University of Berlin Press, 1993

19. Barlow JH, Williams B, Wright C. The generalised self-efficacy scale in people with arthritis. Arthritis Care Res 1996;9:189-96.

20. Horowitz A, Reinhardt JP. Development of the adaptation to age-related vision loss scale. J Vis Impair Blind 1998:92:30-41.

21. Horowitz A, Leonard R, Reinhardt J. Measuring psychosocial and functional outcomes of a group model of vision rehabilitation services for older adults. $J$ Vis Impair Blind 2000:328-37.

22. Hollis $\mathbf{S}$, Campbell $F$. What is meant by intention to treat analysis? Survey of published randomised controlled trials. BMJ 1999;319:670-4.

23. Cohen J. Statistical power analysis for the behavioural sciences. 2nd rev edn. Englewood Cliffs: Lawrence \& Elbaum Associates, 1988.

24. Wang JJ, Foran S, Mitchell P. Age-specific prevalence and causes of bilateral and unilateral visual impairment in older Australians: The Blue Mountains Eye Study. Clin Experiment Ophthalmol 2000;28;268-73.

25. Leinhass MM, Hedstrom NJ. Low vision: how to assess and treat its emotiona impact. Geriatrics 1994;49:53-6. 